\title{
Characterization and structure-activity relationships of indenoisoquinoline-derived topoisomerase I inhibitors in unsilencing the dormant Ube3a gene associated with Angelman syndrome
}

Hyeong-Min Lee ${ }^{1}$, Ellen P. Clark ${ }^{1}$, M. Bram Kuijer ${ }^{1}$, Mark Cushman², Yves Pommier ${ }^{3}$ and Benjamin D. Philpot ${ }^{1,4^{*}}$ (D)

\begin{abstract}
Background: Angelman syndrome (AS) is a severe neurodevelopmental disorder lacking effective therapies. AS is caused by mutations in ubiquitin protein ligase E3A (UBE3A), which is genomically imprinted such that only the maternally inherited copy is expressed in neurons. We previously demonstrated that topoisomerase I (Top 1) inhibitors could successfully reactivate the dormant paternal allele of Ube3a in neurons of a mouse model of AS. We also previously showed that one such Top1 inhibitor, topotecan, could unsilence paternal UBE3A in induced pluripotent stem cell-derived neurons from individuals with AS. Although topotecan has been well-studied and is FDA-approved for cancer therapy, its limited CNS bioavailability will likely restrict the therapeutic use of topotecan in AS. The goal of this study was to identify additional Top1 inhibitors with similar efficacy as topotecan, with the expectation that these could be tested in the future for safety and CNS bioavailability to assess their potential as AS therapeutics.
\end{abstract}

Methods: We tested 13 indenoisoquinoline-derived Top1 inhibitors to identify compounds that unsilence the paternal allele of Ube3a in mouse neurons. Primary cortical neurons were isolated from embryonic day 14.5 (E14.5) mice with a Ube3a-YFP fluorescent tag on the paternal allele (Ube3a ${ }^{m+p Y F P}$ mice) or mice that lack the maternal Ube3a allele and hence model AS (Ube3a ${ }^{m-1 p+}$ mice). Neurons were cultured for 7 days, treated with drug for $72 \mathrm{~h}$, and examined for paternal UBE3A protein expression by Western blot or fluorescence immunostaining. Dose responses of the compounds were determined across a log range of drug treatments, and cytotoxicity was tested using a luciferase-based assay.

Results: All 13 indenoisoquinoline-derived Top1 inhibitors unsilenced paternal Ube3a. Several compounds exhibited favorable paternal Ube3a unsilencing properties, similar to topotecan, and of these, indotecan (LMP400) was the most effective based on estimated $\mathrm{E}_{\max }$ (maximum response of unsilencing paternal Ube3a) and $\mathrm{EC}_{50}$ (half maximal effective concentration).

\footnotetext{
* Correspondence: bphilpot@med.unc.edu

${ }^{1}$ Department of Cell Biology and Physiology, University of North Carolina

School of Medicine, Neuroscience Research Building, Room 5119115 Mason

Farm Rd., Campus Box 7545, Chapel Hill, NC 27599-7545, USA

${ }^{4}$ UNC Neuroscience Center, Carolina Institute for Developmental Disabilities,

University of North Carolina School of Medicine, Chapel Hill, NC, USA

Full list of author information is available at the end of the article
}

(c) The Author(s). 2018 Open Access This article is distributed under the terms of the Creative Commons Attribution 4.0 International License (http://creativecommons.org/licenses/by/4.0/), which permits unrestricted use, distribution, and

reproduction in any medium, provided you give appropriate credit to the original author(s) and the source, provide a link to the Creative Commons license, and indicate if changes were made. The Creative Commons Public Domain Dedication waiver (http://creativecommons.org/publicdomain/zero/1.0/) applies to the data made available in this article, unless otherwise stated. 
(Continued from previous page)

Conclusions: We provide pharmacological profiles of indenoisoquinoline-derived Top1 inhibitors as paternal Ube3a unsilencers. All 13 tested compounds were effective at unsilencing paternal Ube3a, although with variable efficacy and potency. Indotecan (LMP400) demonstrated a better pharmacological profile of Ube3a unsilencing compared to our previous lead compound, topotecan. Taken together, indotecan and its structural analogues are potential AS therapeutics whose translational potential in AS treatment should be further assessed.

Keywords: Angelman syndrome, UBE3A, Topoisomerase I, Topotecan, Indenoisoquinoline, Topoisomerase inhibitor, Indotecan

\section{Background}

Angelman syndrome (AS) is a severe neurodevelopmental disorder characterized by developmental delay, intellectual disability, speech impairment, seizures, and ataxia [1-5]. AS has a prevalence of 1:15,000 [6,7], and these individuals need care across their full lifespan, yet no cure currently exists. Thus, it is of great importance to develop treatments for AS. AS is caused by mutation of the ubiquitin protein ligase E3A (UBE3A) gene, which is genomically imprinted. Only the maternally inherited copy is expressed in neurons [8], whereas $U B E 3 A$ is biallelically expressed in most other tissues. This neuron-specific imprinting provides insight into why deletions or mutations in the maternal copy of $U B E 3 A$ primarily impact brain function and cause AS. However, the paternal $U B E 3 A$ allele is intact, as demonstrated by biallelic expression in other tissues, raising the possibility that AS could be treated by unsilencing the dormant paternal $U B E 3 A$ allele in neurons.

This led us to try pharmacological approaches to identify small molecules capable of unsilencing the dormant copy of $U B E 3 A$. In a previous study, we developed a highcontent assay to identify small molecules that could unsilence paternal $U b e 3 a$ in mouse primary neurons. In that screen, we used knock-in mice carrying a yellow fluorescent protein (YFP)-tagged Ube3a reporter, allowing us to visualize maternal- or paternal-specific expression of Ube3a-YFP in cultured neurons. As expected, Ube3a-YFP was expressed in cultured neurons when inherited maternally but was not expressed (silenced) when inherited paternally. We found that topoisomerase I (Top1) inhibitors (e.g., topotecan) could effectively unsilence paternal Ube3a in mice [9], raising the possibility that topotecan or similar compounds [10] could become treatments for AS. The translational potential was supported by evidence that topotecan treatment biochemically rescued the function of UBE3A, unsilenced $U b e 3 a$ in vivo in mice, and unsilenced paternal $U B E 3 A$ in induced pluripotent stem cell-derived neurons of AS patients [11].

Topotecan is FDA-approved for the treatment of cancer and is well tolerated in adult and pediatric cancer patients [12-15]. It is also used to treat brain tumors [16, 17]. Topotecan crosses the BBB more readily than many topoisomerase inhibitors [18]. However, active pumps extrude topotecan from the brain, which limits its functional CNS bioavailability $[19,20]$. Moreover, topotecan can produce some toxicities [21, 22]. These limitations prompted us to search for novel Top1 inhibitors with better CNS bioavailability and improved safety profiles.

Indenoisoquinoline-derived Top1 inhibitors offer a promising class of compounds for paternal Ube3a unsilencing as many of these compounds produce particularly stable Top1 cleavage complexes [23-25], which we have shown are critical for producing paternal Ube3a unsilencing [26]. Over 300 indenoisoquinoline derivatives have been tested, some of which are very potent Top1 poisons and show antitumor activity in mouse models [10, 27-31]. These Top1 inhibitors work by blocking the enzymatic activity of Top1 by stabilizing cleavage complexes, which are compound-bound intermediates of Top1-DNA [10, 23-25, 32]. More importantly, when compared to topotecan, indenoisoquinoline-derived Top1 inhibitors demonstrate improved characteristics such as greater chemical stability of these cleavage complexes. In addition, they target a unique DNA sequence for cleavage (indenoisoquinolines -- $\mathrm{G}^{\downarrow} \mathrm{C}-$ - vs. topotecan $\left.-\mathrm{T}^{\downarrow} \mathrm{G}--\right)[23-25,32]$.

The goal of this study was to establish indenoisoquinoline derivatives that could effectively unsilence paternal Ube3a, with the expectation that some of these compounds might prove to be safe and have favorable CNS bioavailability. All of the tested compounds showed a capacity to unsilence the paternal Ube3a allele, with several of the compounds exhibiting unsilencing efficacy similar to topotecan. Excitingly, two of the tested indenoisoquinoline derivatives, indotecan (LMP400) and indimitecan (LMP776), are already in clinical trials [33, 34]. The results of our study suggest additional Top 1 inhibitors that should be advanced for AS preclinical testing of safety and CNS efficacy.

\section{Methods}

\section{Animals}

All animal experiments were handled with an Institutional Animal Care and Use Committee (IACUC) protocol approved by the University of North Carolina School of Medicine. AS model mice [35] $\left(U b e 3 a^{m-/ p+}\right)$ were 
generated by crossing $U b e 3 a^{m+/ p-}$ females with wildtype males. Paternal YFP-tagged mice [36] $\left(U b e 3 a^{\mathrm{m}+/ p Y F P}\right)$ were generated by crossing heterozygote Ube3a-YFP males with wildtype females. Mice were housed at $12 \mathrm{~h}: 12 \mathrm{~h} \mathrm{LD}$ and given ad libitum access to water and food. Both male and female mice (embryos) were used in all studies.

\section{Chemistry}

Topotecan was purchased from Cayman Chemicals. Indotecan (LMP400) and indimitecan (LMP776) were obtained from the Developmental Therapeutics Program (DTP) branch, National Cancer Institute. Eleven structural analogues of indotecan and indimitecan, all indenoisoquinoline derivatives, were provided by Dr. Mark Cushman at Purdue University. Syntheses of 12 indenoisoquinolines have been previously described: indotecan (LMP400) and indimitecan (LMP776) [33], DB-III-17, DB-IV-26, DB-IV-50, DB-IV-56, DB-IV-58, DB-V-37, DB-V-41, DB-V-46, and DB-V-47 [37], and MJ-II-66A [30]. MNR-IV-64 was synthesized by the procedure reported for the corresponding $N-4^{\prime}$-hydroxybutyl analogue [30]. These compounds were selected because they have a wide range of cytotoxicities in cancer cell culture while maintaining some degree of Top1 inhibitory activity. All compounds were stored at $-20^{\circ} \mathrm{C}$ before being reconstituted in dimethyl sulfoxide (DMSO) prior to use.

\section{Cell culture and drug treatment}

Primary cortical neurons were isolated and cultured using previously described protocols [9]. Briefly, we isolated cortical neurons from embryos (E14.5) carrying paternal Ube3a-YFP $\left(U b e 3 a^{\mathrm{m}+/ p Y F P}\right)$ or maternal deletion of Ube3a $\left(\right.$ Ube $\left.3 a^{\mathrm{m}-/ p+}\right)$. Isolated cortical neurons were plated onto 384-well plates $(\sim 25,000$ cells/well $)$ for high-content imaging and onto 6 -well plates $(\sim 1,000,000$ cells/well $)$ for Western blot analysis. Cultured neurons were initially incubated for 7 days, replacing culture medium every 3-4 days. Drugs were freshly prepared in DMSO as a $10-\mathrm{mM}$ stock, unless further dilution in DMSO was necessary. On day 7 (DIV 7), the indicated compounds (topotecan, indotecan, indimitecan, DB-IV-58, and DB-V-37 in Figs. 1, 2, and 3) were directly added at $0.3 \mu \mathrm{M}$ (final concentration in culture medium) to the neurons for $72 \mathrm{~h}$ to allow time for unsilencing of paternal Ube3a or Ube3a-YFP. For dose dependence and cytotoxicity tests (Fig. 4 and Additional file 1), we used half-log molar drug concentrations, $1 \times 10^{-10}, 3 \times$ $10^{-10}, 1 \times 10^{-9}, 3 \times 10^{-9}, 1 \times 10^{-8}, 3 \times 10^{-8}, 1 \times 10^{-7}, 3 \times$ $10^{-7}, 1 \times 10^{-6}, 3 \times 10^{-6}, 1 \times 10^{-5}$, and $3 \times 10^{-5} \mathrm{M}$.
A
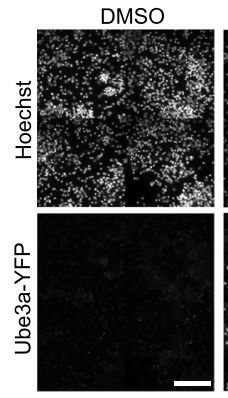

B

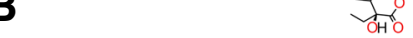

Indotecan (LMP400)
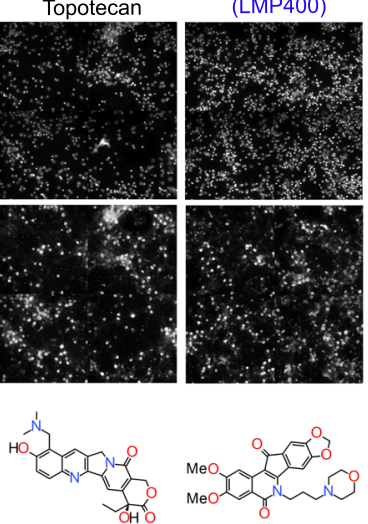

Indimitecan
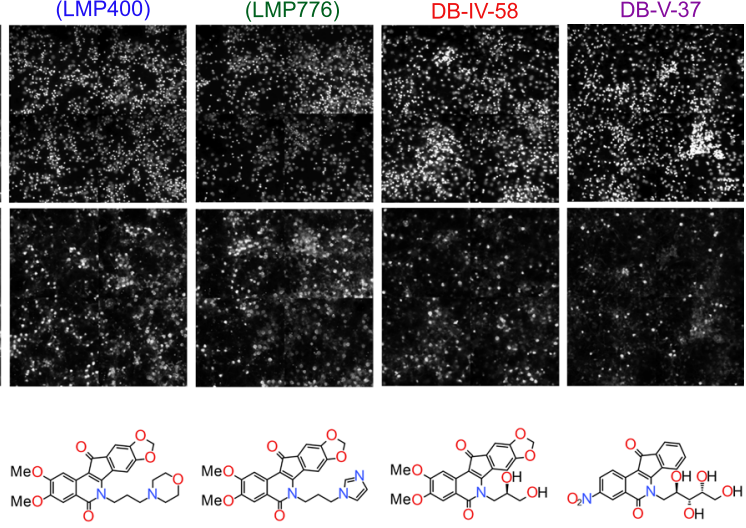

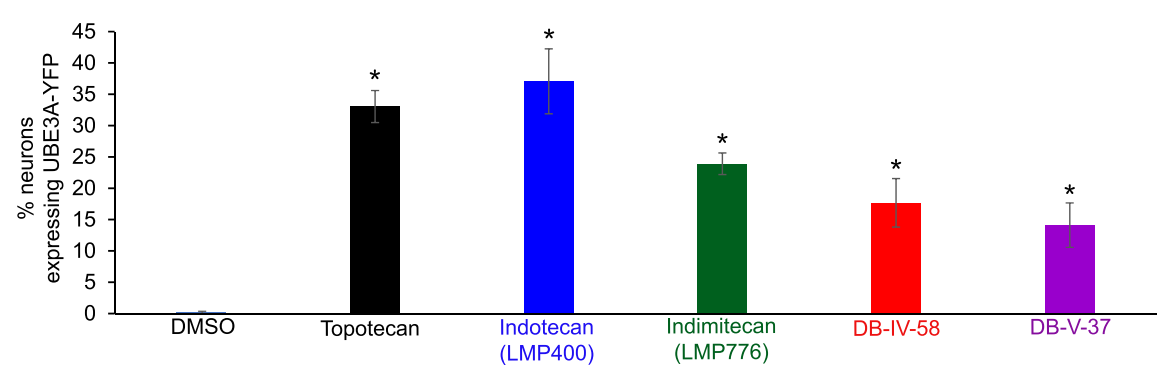

Fig. 1 Like the camptothecin-derived topotecan, indenoisoquinoline derivatives can unsilence paternal Ube3a-YFP in vitro. a Immunofluorescence images of nuclei (Hoeschst stain) and paternal UBE3A-YFP in drug-treated cultured mouse cortical neurons and chemical structures of the compounds. Paternal Ube3a-YFP was unsilenced by the indicated drugs [topotecan $(0.3 \mu \mathrm{M})$, indotecan $(0.3 \mu \mathrm{M})$, indimitecan $(0.3 \mu \mathrm{M})$, DB-IV-58 (0.3 $\mu \mathrm{M})$, or DB-V-37 (0.3 $\mu \mathrm{M})$ ] but not by DMSO vehicle control (scale bar $=100 \mu \mathrm{m}$ ). DB-IV-58 and DB-V-37 are structural analogues of indotecan and indimitecan. $\mathbf{b}$ Quantitative analysis of neurons expressing unsilenced paternal UBE3A-YFP ( $n=4$ wells in 384-well plate/group, ${ }^{*} p<0.05$ ) 
A
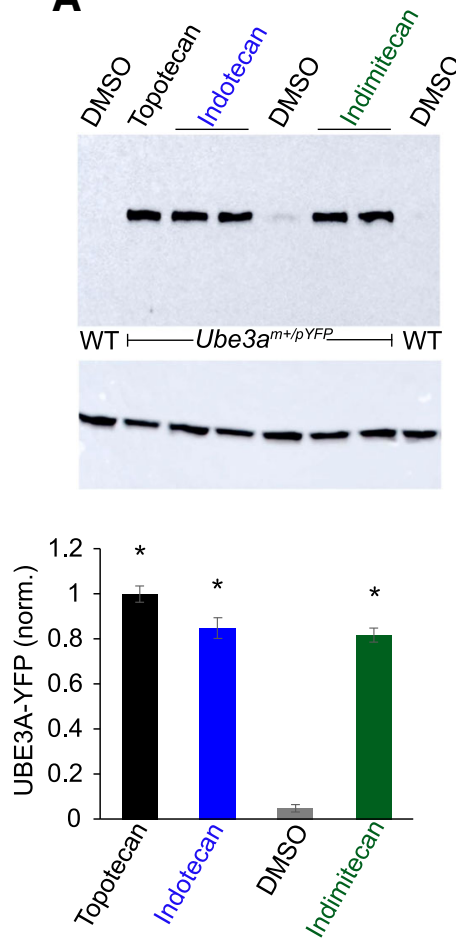

B

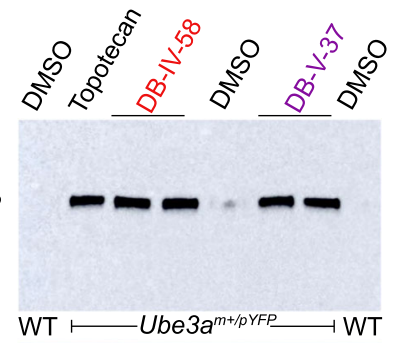

Actin
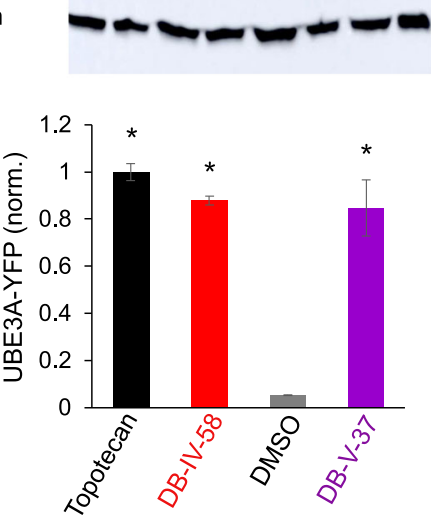

Fig. 2 Western blot analysis demonstrating the capacity of indenoisoquinoline derivatives to increase paternal UBE3A-YFP at the protein level in cultured neurons from Ube3a ${ }^{\mathrm{m}+/ \text { PYFP }}$ mice. a Immunoblot and quantification of UBE3A-YFP levels normalized to actin in cultured neurons from wildtype (WT) or Ube $3 a^{\mathrm{m}+/ p Y F P}$ mice treated with DMSO $\left(0.1 \%\right.$ vehicle control), topotecan $(0.3 \mu \mathrm{M})$, indotecan $(0.3 \mu \mathrm{M})$, or indimitecan $(0.3 \mu \mathrm{M})\left(n=3 / \mathrm{group}\right.$, $\left.{ }^{*} p<0.05\right)$. $\mathbf{b}$ Immunoblots and quantification of UBE3A-YFP levels normalized to actin in cultured neurons from wildtype (WT) or Ube3am+/pYFP mice treated with DMSO (0.1\% vehicle control), topotecan $(0.3 \mu \mathrm{M})$, DB-IV-58 $(0.3 \mu \mathrm{M})$, or DB-V-37 $(0.3 \mu \mathrm{M})\left(n=3 /\right.$ group, $\left.{ }^{*} p<0.05\right)$

Fluorescence immunostaining and high-content imaging We followed the protocols for fluorescent immunostaining and high-content imaging of cortical neurons as previously described [9, 38]. Briefly, $72 \mathrm{~h}$ after drug treatment, the cells were fixed with $4 \%$ paraformaldehyde at room temperature for $15 \mathrm{~min}$. After rinsing with phosphate-buffered saline (PBS) three times, the cells were permeabilized with $1 \%$ Triton-X 100 in PBS, followed by blocking with $5 \%$ NGS and $0.1 \%$ Triton-X 100 in PBS at room temperature for $30 \mathrm{~min}$. After blocking, the cells were incubated with primary antibody, rabbit anti-GFP (1:1000, Novus Biologicals), at $4{ }^{\circ} \mathrm{C}$ overnight. The cells were then briefly rinsed with PBS followed by incubation with secondary antibody, goat anti-rabbit Alexa Fluor 488 (1:200, Thermo Fisher/Invitrogen), at room temperature. One hour after secondary antibody incubation, the cells were rinsed with PBS and fluorescent images were acquired using a BD Pathway 855 bioimager. The acquired images were processed by CellProfiler [39] to count the number of positive cells and measure fluorescent intensity (Additional file 2). To determine the percentage of neurons expressing paternal Ube3a-YFP, we counted the total number of cells (Hoechst) and YFP-positive cells, and the number of
YFP-positive cells was divided by the total number of cells. Fluorescence intensity was measured in neurons expressing unsilenced paternal Ube3a-YFP and normalized to vehicle control.

\section{Cytotoxicity test}

Toxicity of the compounds was tested in cultured cortical neurons in vitro. Using Cyto Tox-Glo assay (Promega), we followed the manufacturer's protocol to measure luminescence proportional to the number of dead vs. live cells. Briefly, $72 \mathrm{~h}$ after drug treatment, we directly added AAF-Glo substrates into the drug-treated (or 0.1\% DMSO vehicle-treated) neurons and incubated them at room temperature for $15 \mathrm{~min}$. We then measured luminescence produced by dead-cell protease activity.

\section{Western blot analysis}

We followed the same procedures of Western blot analysis as previously described [9, 38]. In brief, $72 \mathrm{~h}$ after drug treatment, we collected the cultured neurons from 6-well plates and extracted total protein with protein extraction buffer. Bradford assay was performed to measure protein concentration, and $30 \mu \mathrm{g}$ of total protein was loaded for Bis-polyacrylamide gel electrophoresis 


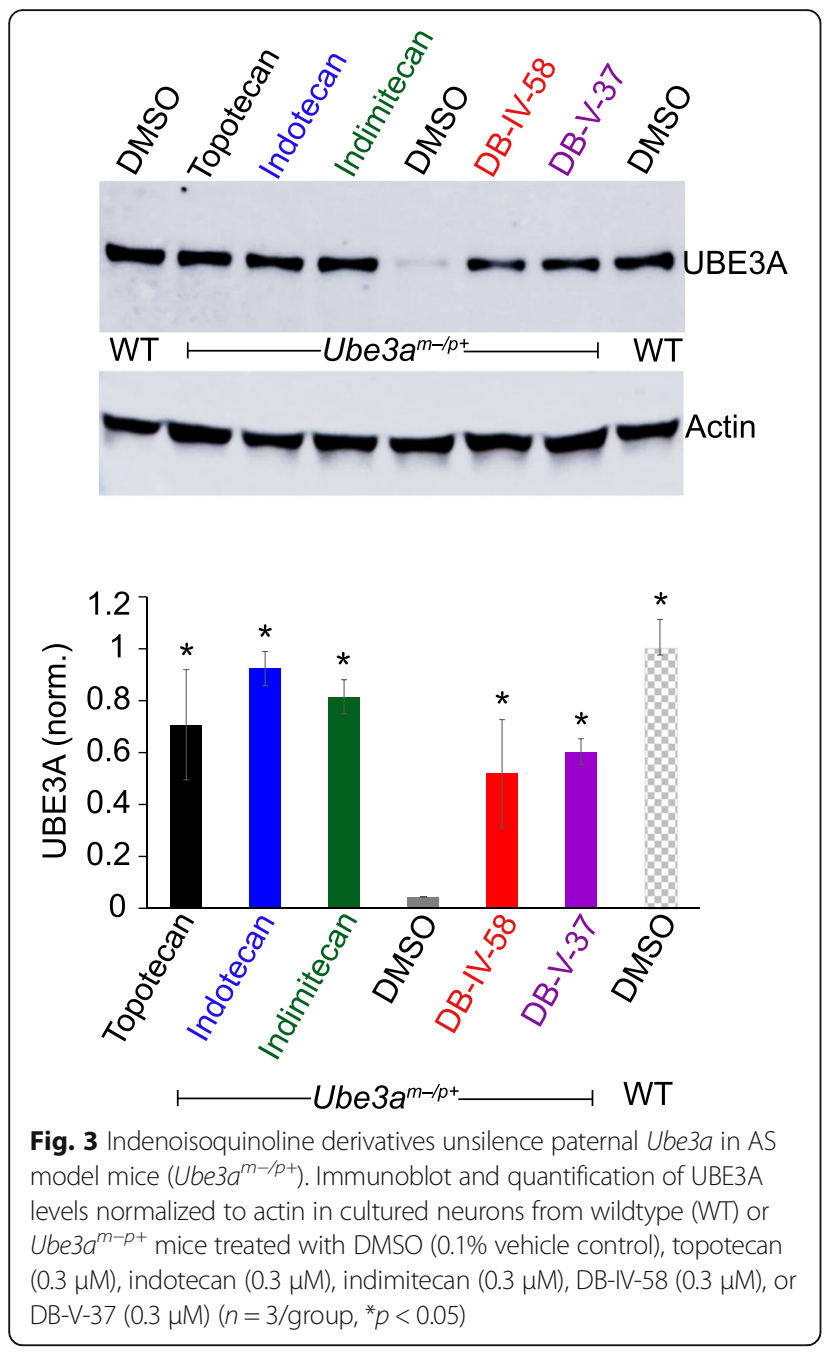

(Bio-Rad). Electrophoresed proteins were transferred to nitrocellulose membrane $(0.45 \mu \mathrm{m}$, Bio-Rad). The membranes were blocked with $5 \%$ non-fat milk in TBS-T at room temperature for $30 \mathrm{~min}$ followed by overnight $4{ }^{\circ} \mathrm{C}$ incubation with primary antibodies (rabbit anti-GFP, 1:1000, Novus Biologicals; rabbit anti-Ube3a, 1:1000, Bethyl Lab; mouse anti-actin; 1:5000, Sigma). The next day, the membranes were rinsed with TBS-T three times and incubated with HPR-conjugated secondary antibodies for $1 \mathrm{~h}$ at room temperature (goat anti-rabbit, 1:1000, Vector Lab or goat anti-mouse, 1:1000, Vector Lab). Following secondary antibody incubation, the membranes were rinsed with TBS-T at room temperature for $1 \mathrm{~h}$ (4-5 times) and ECL substrates (Bio-Rad) were used to visualize immunostaining using an Amersham Imager 600 (AI600, GE Life Sciences).

\section{Statistical analysis}

One-way ANOVA was performed to determine significant differences in unsilencing paternal Ube3a-YFP or Ube3a, followed by Dunnett's multiple comparison test.
Two-way ANOVA was performed to determine changes in $\mathrm{EC}_{50}, \mathrm{E}_{\max }$, and $\mathrm{LC}_{50}$ (Table 1), with comparisons to topotecan made using a Bonferroni correction for multiple comparisons.

\section{Results}

Indenoisoquinoline-derived topoisomerase I inhibitors effectively unsilence paternal Ube3a

The goal of this study was to identify novel Top1 inhibitors as potential AS therapeutics. We chose to focus on the compounds indotecan (LMP400), indimitecan (LMP776), and their analogues because indotecan and indimitecan recently completed phase I clinical trial testing at the National Institutes of Health (ClinicalTrials.gov ID: NCT01051635). Moreover, indotecan exhibits favorable CNS penetration [40]. Thus, these drugs have already undergone a certain degree of preclinical safety testing, which could expedite clinical development if warranted.

As we anticipated, paternal Ube3a-YFP was not expressed at appreciable levels in cultured neurons in the presence of $0.1 \%$ DMSO (vehicle control). On the other hand, topotecan (positive control) unsilenced paternal Ube3a-YFP as previously reported [9] (Fig. 1a). Four indenoisoquinolinederived compounds (indotecan, indimitecan, DB-IV-58, and DB-V-37) also successfully demonstrated unsilencing of paternal Ube3a-YFP in our reporter mouse (Fig. 1a). We quantified the number of Hoechst-positive cells expressing paternal Ube3a-YFP above a defined threshold and found that few $(0.21 \pm 0.17 \%)$ DMSO-treated neurons expressed Ube3a-YFP above threshold (Fig. 1b). In contrast, a significant $(p<0.05)$ number of neurons expressed paternal Ube3a-YFP when cultures were treated with topotecan (33.0 $\pm 2.56 \%)$, indotecan $(37.1 \pm 5.19 \%)$, indimitecan (23.9 $\pm 1.72 \%)$, DB-IV-58 (17.7 $\pm 3.87 \%)$, or DB-V-37 (14.1 \pm 3.55\%) (Fig. 1b). The number of cells expressing paternal Ube3a-YFP was similar between indotecan- and topotecantreated neurons at a dose of $0.3 \mu \mathrm{M}$. At this same concentration $(0.3 \mu \mathrm{M})$, fewer neurons treated with indimitecan, DB-IV-58, or DB-V-37 expressed paternal Ube3a-YFP compared to topotecan-treated neurons $(p<0.05$ compared to topotecan-treated neurons).

To validate the paternal Ube3a-YFP unsilencing and definitively rule out the possibility of fluorescence artifacts (e.g., intrinsic fluorescence in compounds), we performed Western blot analysis using primary cultured cortical neurons. No UBE3A-YFP protein was expressed in wildtype neurons (negative control), whereas paternal UBE3A-YFP was marginally detectable in DMSO-treated cells from $U b e 3 a^{m+/ p Y F P}$ mice, possibly due to contamination from glial cells that express Ube3a biallelically or to very modest expression of paternal Ube3a expressed during early stages of development. Topotecan, indotecan, indimitecan, DB-IV-58, and DB-V-37 treatments led to paternal UBE3A-YFP protein production (Fig. 2). Normalized 

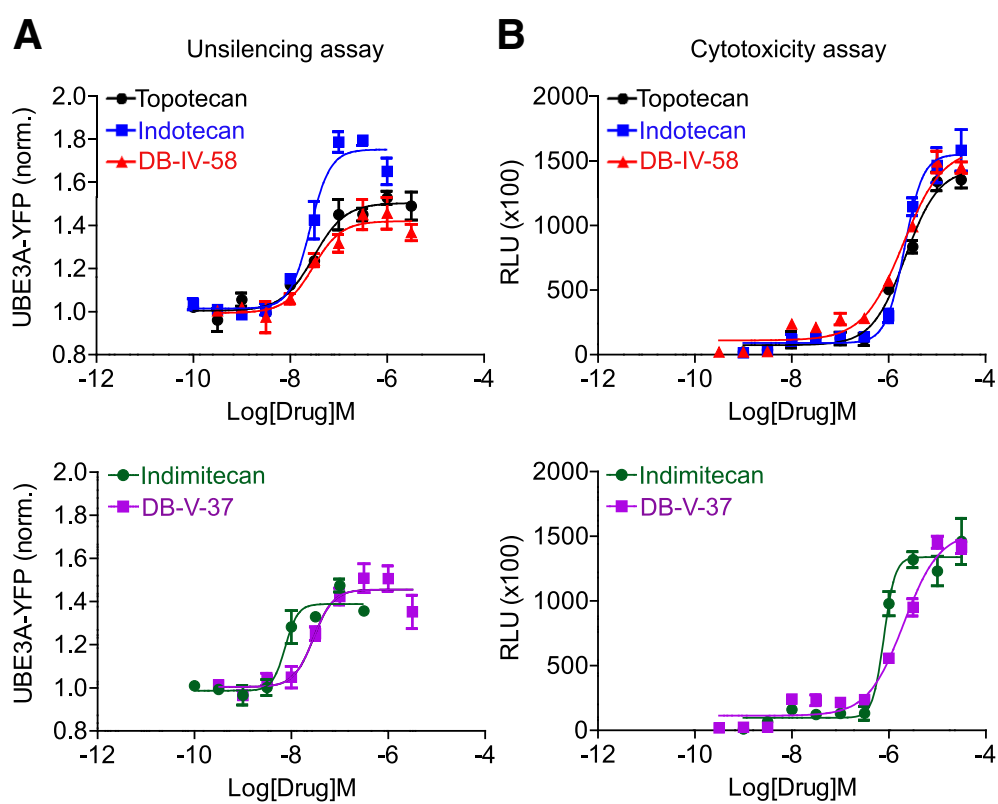

Fig. 4 Pharmacological properties of four indenoisoquinoline derivatives in unsilencing paternal Ube3a-YFP in vitro. a Dose dependence of four indenoisoquinoline derivatives and topotecan in unsilencing of paternal UBE3A-YFP ( $n=4 /$ group). Estimated potencies and efficacies of the drugs are summarized in Table 1. $\mathbf{b}$ Dose-dependent cytotoxicities of four indenoisoquinoline derivatives and topotecan $(n=4 / \mathrm{group})$. Estimated $L C_{50}$ values are summarized in Table 1

fold changes in unsilenced UBE3A-YFP were comparable in all five tested drugs (bottom panels in Fig. 2a, b).

Although unlikely, we wanted to rule out the possibility that the unsilencing was an artifact of the Ube3a-YFP knock-in. Thus, we tested the drug effects in AS model mice that lack the maternal Ube3a allele (Ube3 $a^{m-1 p+}$ mice) (Fig. 3). There was little paternal UBE3A protein in DMSO-treated Ube3a $a^{m-p+}$ neurons compared to Ube3a $a^{m+1 p+}$ (wildtype) neurons. Topotecan, indotecan, indimitecan, DB-IV-58, and DB-V-37 treatments result in a high level of paternal UBE3A protein compared to DMSO-treated neurons. These data confirm the ability of the tested indenoisoquinolines to unsilence paternal Ube3a.

\section{Pharmacological profiling of indenoisoquinoline Top1 inhibitors in cultured cortical neurons in vitro}

Once we confirmed the unsilencing effects, we performed pharmacological profiling of indotecan, indimitecan, and their analogues for structure-activity relationships in order to identify more effective unsilencers. We performed dose-response experiments for all tested compounds (Fig. 4a and Additional file 1). These experiments identified indotecan as the most efficacious of all of drugs tested, with the potency $\left(\mathrm{EC}_{50}\right)$ of indotecan being significantly better than topotecan (Table $1 ;{ }^{*} p<0.05$ ). This suggests indotecan may have potential as a possible AS therapeutic. Indimitecan is less likely as a candidate therapeutic, because although it exhibited good potency in a nanomolar range and had similar efficacy as topotecan (Fig. 4a and Table 1, ${ }^{*} p<0.05$ ), it likely has a low therapeutic index (discussed below). We also examined 11 structural analogues. Among 11 compounds, we found two of them, DB-IV-58 and DB-V-37, with similar efficacy and potency to topotecan (Fig. 4a and Table 1). However, they were less effective than indotecan.

Indenoisoquinoline derivatives were primarily designed to inhibit cancer cell growth. Because our goal is to

Table 1 Summary of efficacies and potencies of topotecan and indenoisoquinoline derivatives

\begin{tabular}{llll}
\hline Compound & $\mathrm{EC}_{50}[\mathrm{M}]$ & $\mathrm{E}_{\max }$ & $L C_{50}[\mathrm{M}]$ \\
\hline Topotecan & $3.04 \mathrm{E}-08( \pm 3.40 \mathrm{E}-09)$ & $1.51( \pm 0.11)$ & $2.30 \mathrm{E}-06( \pm 2.01 \mathrm{E}-08)$ \\
Indotecan & $2.56 \mathrm{E}-08( \pm 1.05 \mathrm{E}-09)^{*}$ & $1.78( \pm 0.13)^{*}$ & $2.01 \mathrm{E}-06( \pm 1.59 \mathrm{E}-07)$ \\
Indimitecan & $7.47 \mathrm{E}-09( \pm 7.49 \mathrm{E}-10)^{*}$ & $1.47( \pm 0.10)$ & $7.89 \mathrm{E}-07( \pm 3.43 \mathrm{E}-08)^{*}$ \\
DB-IV-58 & $3.10 \mathrm{E}-08( \pm 9.75 \mathrm{E}-09)$ & $1.45( \pm 0.12)$ & $2.01 \mathrm{E}-06( \pm 1.09 \mathrm{E}-07)$ \\
DB-V-37 & $2.99 \mathrm{E}-08( \pm 8.29 \mathrm{E}-09)$ & $1.48( \pm 0.08)$ & $2.02 \mathrm{E}-06( \pm 1.02 \mathrm{E}-07)$ \\
\hline
\end{tabular}

$\mathrm{EC}_{50}, \mathrm{E}_{\mathrm{max}}$ and $\mathrm{LC}_{50}$ are estimated from data presented in Fig. 4. Significance was tested by two-way ANOVA, followed by a Bonferroni correction for multiple comparisons to determine significant differences $\left({ }^{*} p<0.05\right)$ from topotecan-treated neurons 
repurpose these drugs to unsilence a CNS target, it was necessary to test whether the compounds would be deleterious in neuronal cells. Toxicity testing of four indenoisoquinoline-derived compounds (indotecan, DB-IV-58, DB-V-37, and indimitecan) in the cultured neurons revealed that the cytotoxicity of the first three drugs (indotecan, DB-IV-58, and DB-V-37) was similar to that of topotecan. On the other hand, indimitecan exhibited toxicity at a significantly lower concentration than topotecan (Fig. 4b and Table 1, $* p<0.05)$, suggesting that it might have a low therapeutic index. Lastly, we also tested dose dependency of nine additional analogues (Additional file 1 and Additional file 3: Table S1). All nine compounds could unsilence paternal Ube3a-YFP to a certain degree. However, their effectiveness did not exceed that of indotecan. Three compounds (DB-IV-50, DB-IV-56, and MNR-IV-64) share similar pharmacological profiles, as their efficacy and potency were similar. However, their effectiveness of unsilencing paternal Ube3a-YFP was less than our lead compounds. Six compounds (DB-III-17, DB-IV-26, DB-V-41, DB-V-46, DB-V-47, and MJ-II-66A) unsilenced paternal Ube3a-YFP to a certain degree; however, they showed a very limited range of doses that produce Ube3a-YFP unsilencing before showing toxicities (e.g., an unfavorable therapeutic index). Because of the limited dose ranges, their $\mathrm{EC}_{50}$ values were not clearly determined (Additional file 3: Table S1). These data together suggest that, compared to topotecan, indotecan has a higher potency and efficacy, but similar toxicity profile, in its ability to unsilence the dormant paternal Ube3a allele in neurons.

\section{Discussion}

The goal of this study was to explore indenoisoquinoline derivatives as possible AS therapeutics by characterizing their effects on Ube3a unsilencing in mouse cortical neurons in vitro. Here, we identify indotecan (LMP400) as a potential AS therapeutic agent that warrants further examination in vivo for CNS bioavailability and safety.

The unique expression of $U B E 3 A$ governed by genomic imprinting provides a therapeutic opportunity for AS by reactivating the paternal $U B E 3 A$ allele $[8,9,11]$. Our research team previously reported that topoisomerase I inhibitors can reactivate the dormant $U B E 3 A$ allele, providing the first proof of concept of pharmacological reactivation of paternal $U B E 3 A$ as a potential therapeutic intervention for AS [9]. Because of the anticancer activity of Top1 inhibitors, many derivatives that overcome the limitations of camptothecins [10, 41, 42] have been synthesized for clinical development. One of these, topotecan, is FDA-approved for ovarian and lung cancers [43], while another, irinotecan, is approved for colon cancers [44]. However, these compounds may have limited clinical potential for treating AS. For example, topotecan has several flaws such as decreased bioavailability due to plasma protein binding of the lactone hydrolysis product, removal from cells by drug efflux transporters, and long infusion times necessitated by relatively low stability of the ternary drug-DNA-enzyme cleavage complexes [10, 18]. These limitations prompted us to search for novel Top1 inhibitors as potential AS therapeutics, with the expectation that lead candidates could then be vetted for having improved CNS bioavailability and better safety profiles. For these studies, we focused on indenoisoquinoline-derived Top1 inhibitors that might overcome the limitations of topotecan [10]. Of many indenoisoquinoline-derived Top1 inhibitors, indotecan and indimitecan were selected to examine their unsilencing effects on paternal Ube3a because of their similar ability to effectively inhibit almost $100 \%$ of Top1 enzymatic activity [33] and recent completion of phase I clinical trials (ClinicalTrials.gov ID: NCT01051635).

Here, we demonstrate that indenoisoquinoline-derived Top1 inhibitors are potent $U b e 3 a$ unsilencers, with different unsilencing properties. Of the compounds we tested in vitro, indotecan (LMP400) [10, 34] appears to have more favorable paternal $U b e 3 a$ unsilencing properties than topotecan. While both topotecan and indotecan exhibit CNS penetrance [18, 40], there are not yet data available to directly compare their relative CNS bioavailability; there is a need to carefully establish the CNS bioavailability of indotecan. One potential advantage of indotecan is that it is not a substrate for the transporters ABCG2 and MDR1 [32], suggesting that it may stay longer in the CNS than topotecan because transporters extrude topotecan from the brain [19]. Moreover, although the cytotoxicity profile of indotecan is similar to that of topotecan, its efficacy and potency are better than topotecan. Indimitecan (LMP776) [10] appears to be more toxic than topotecan, while the three other indenoisoquinoline derivatives (indotecan, DB-IV-58, and DB-V-37) showed similar cytotoxicity to topotecan in our cultured cortical neurons. In addition, the efficacy of indimitecan is lower than that of topotecan. The structural differences in indenoisoquinoline-derived compounds are responsible for their different unsilencing effects. The only structural difference between indotecan and indimitecan is in the side chain which is appended to the heterocyclic system that intercalates in the DNA break generated by Top1 [10, 23]. These characteristics will be important considerations for the future design of paternal $U B E 3 A$ unsilencers. Although indotecan exhibits better efficacy and potency than topotecan, the similar cytotoxicity of the two drugs must be considered for in vivo applications. Importantly, the DNA cleavage complex patterns of indotecan are different from topotecan in a manner that may confer some important advantages for clinical use. Indenoisoquinolines such as 
indotecan produce more stable cleavage complexes than camptothecins such as topotecan [45], which, based on the mechanism of Ube3a unsilencing [26], should enhance Ube3a unsilencing as we observed. Moreover, after drug removal, the Top1-DNA complexes induced by indenoisoquinolines persist under conditions where camptothecin-induced Top1-DNA complexes completely reverse [45]. This observation further suggests that the similar cytotoxicity of indotecan might be offset by the potential for a briefer treatment regimen in vivo, which remains to be addressed. Known off-target effects for topoisomerase inhibitors generally lead to the transient downregulation of long genes [11, 46, 47]. Genomewide analyses are necessary to reveal all potential offtarget effects for indenoisoquinolines (e.g., indotecan). Alternatively, an in silico analysis using SEA (similarity ensemble approach; http://sea.bkslab.org) enables us to predict off-targets. SEA analysis revealed that indotecan possesses 55 potential off-targets, including aurora kinase A, aurora kinase B, and acetylcholinesterase. Regardless of off-target effects, clinical trials have demonstrated that, at least at the concentrations examined, indotecan is well tolerated in a clinical population [34]. However, it still remains to be addressed whether off-target effects arise at the concentration at which indotecan is effective, as we reported that indotecan has a very low $\mathrm{EC}_{50}$ of $\sim 26 \mathrm{nM}$ to produce Ube3a unsilencing.

We tested 11 structural analogues of indotecan/indimitecan for their ability to unsilence paternal $U b e 3 a$, and these compounds could be roughly categorized based on their ability to inhibit Top1 in cell-free assays: those compounds (DB-IV-26, DB-IV-50, DB-IV-56, DB-IV-58, DB-V-37, and DB-V-41) that have $100 \%$ of the ability of camptothecin to stabilize the ternary drug-DNA-Top1 cleavage complexes [37, 48], those compounds (DB-V-46, DB-V-47, and MNR-IV-64) that inhibit between 50 and 75\% of Top1 [37], and those compounds (MJ-II-66A) that inhibit between 20 and $50 \%$ of Top1 [30]. We also tested DB-III-17, as this is an intermediate compound for synthesizing or modifying the analogues. The compounds with lower Top1 inhibitory activities (DB-III-17, DB-V-46, DB-V-47, and MJ-II-66A) showed a very limited therapeutic index. Their ambiguous $\mathrm{EC}_{50}$ values were mainly due to limited effective dose ranges. Although we did not test their cytotoxicities in our cultured cortical neurons, we expect that they are more toxic than topotecan because we could not measure the fluorescence intensity in unsilenced UBE3A-YFP protein, possibly due to cell death produced at concentrations $>1 \mu \mathrm{M}$. On the other hand, we observed similar efficacy and potency of three compounds (DB-IV-50, DB-IV-56, and MNR-IV-64) with between 75 and $~ 100 \%$ Top1 inhibitory activities relative to camptothecin, but their effectiveness seems to be less than those of indotecan or topotecan. Interestingly, although DB-IV-26 and
DB-V-41 have 100\% Top1 inhibitory activity, their EC50 values were also ambiguous due to limited effective dose ranges. Since the cleavage complexes are critical for producing paternal Ube3a unsilencing [26], we suspect that their cleavage complexes may not be stable enough to produce paternal Ube3a unsilencing. More importantly, the various hydroxylated side chains contribute to differences in the pharmacological action in Ube3a unsilencing. For example, the compounds with lower Top1 inhibitory activities (DB-III-17, DB-V-46, DB-V-47, and MJ-II-66A) either lack the hydroxylated side chains that potentially serve as hydrogen-bond acceptors/donors that enable Top1 inhibitory activities and cytotoxicity at physiological $\mathrm{pH}$ [49] or the hydroxylated side chain is cyclic. On the other hand, other compounds possessing dimethoxy or methylenedioxy groups, and/or straight hydroxylated side chains, which appear to be the main contributors to Top1 inhibitory activity and cytotoxicity, effectively unsilence paternal Ube3a.

Taken together, our study suggests that clinical development of paternal Ube3a unsilencers will require optimization of Top1 inhibition and cytotoxicity through modulating chemical characteristics, including the length of the side chains. Although in vivo assays are necessary to further evaluate the unsilencing effects of indenoisoquinolines, this study provides a framework for developing novel AS therapies using different classes of Top1 inhibitors.

\section{Conclusions}

Angelman syndrome is a neurodevelopmental disorder without effective therapeutic interventions. However, pharmacological restoration of the epigenetically silenced copy of $U B E 3 A$ could be one promising approach. Pharmacological inhibition of topoisomerase I (Top1) leads to re-expression of the dormant $U B E 3 A$ allele. Here, we provided pharmacological profiles of indenoisoquinolinederived Top1 inhibitors as Ube3a unsilencers in mouse neurons to identify potential AS clinical candidates. Our data suggest that a number of indenoisoquinolines, and in particular indotecan (LMP400), are potent unsilencers of the paternal $U b e 3 a$ allele and should be further assessed in vivo for their translational potential.

\section{Additional files}

Additional file 1: Chemical structures of nine analogues of indotecan/ indimitecan and their pharmacological properties in unsilencing of paternal UBE3A-YFP in vitro. A. DB-III-17, B. DB-IV-26, C. DB-IV-50, D. DB-IV56, E. DB-V-41, F. DB-V-46, G. DB-V-47, H. MJ-II-66A, I. MNR-IV-64. Estimated potencies and efficacies of the drugs are summarized in Additional file 3: Table S1. (DOCX 13 kb)

Additional file 2: Image analysis in CellProfiler. Morphological changes in the neurons were assessed by nuclear structure of Hoechst-stained neurons. A Immunofluorescence images of Hoechst-stained neurons obtained using 
a BD Pathway 855 high-content imager (top). The size (between 18 and 40 pixel units) and intensity (threshold range between 0.005 and 1) of stained nuclei were used to segregate putative viable cells recognized as objects (middle, green) from clumped or dead cells (middle, red). Recognized objects were further processed based on size, intensity, and shape (round) (bottom, individual objects were assigned colors by CellProfiler to allow them to be better visualized). No objects were identified in the neurons treated with $10 \mu \mathrm{M}$ indotecan, as no nuclei met the criteria of immunofluorescence size and intensity. B Quantitative analysis of identified objects. The average numbers of objects were comparable between neurons treated with DMSO $(0.1 \%$ vehicle control) and indotecan $(0.01 \mu \mathrm{M}$ and $0.3 \mu \mathrm{M})$-treated neurons. (PDF $338 \mathrm{~kb}$ )

Additional file 3: Table S1. Potency and efficacy of nine analogues (PDF $270 \mathrm{~kb}$ )

\section{Abbreviations}

AAF-Glo: Alanyl-alanyl-phenylalanyl-aminoluciferin; AS: Angelman syndrome; BBB: Blood-brain barrier; CNS: Central nervous system; DIV: Day in vitro; FBS: Fetal bovine serum; LMP: Laboratory of Molecular Pharmacology at National Cancer Institutes; NGS: Normal goat serum; PBS: Phosphate buffer saline; PBS-T: Phosphate buffer saline Triton X-100; Ube3a: Ubiquitin protein ligase E3A; Ube3a $a^{m-1 p+}$ : Maternal deletion of ubiquitin protein ligase E3A and intact paternal Ube3a; Ube3a ${ }^{m+/ p Y F P}$ : Intact maternal Ube3a and paternal yellow fluorescence protein (YFP)-tagged Ube3a

\section{Acknowledgements}

We would like to thank the Developmental Therapeutics Program (DTP) branch, National Cancer Institute, for providing indotecan (LMP400) and indimitecan (LMP776). We would like to thank Erika Wittchen, PhD, for proofreading the manuscript.

\section{Funding}

The work was supported by NINDS R01NS085093 and a grant from the Angelman Syndrome Foundation to BDP. The research at Purdue was facilitated by the National Institutes of Health through support with Research Grant P30CA023168. YP's research was supported by the Center for Cancer Research, the Intramural Program of the National Cancer Institute (NIH) (Z01-BC006161).

\section{Availability of data and materials}

The pharmacological datasets are available to academic researchers upon request. Indotecan (LMP400) and indimitecan (LMP556) are available from the Developmental Therapeutics Program (DTP) branch, National Cancer Institute (NCl).

\section{Authors' contributions}

HML designed and performed all experiments. EPC and MBK assisted and supported cell culture and neuronal isolation. MS and YP provided the compounds tested in this study. The manuscript was written by HML, MC, YP, and BDP. All authors reviewed and agreed to submit the final manuscript.

\section{Ethics approval and consent to participate}

Not applicable

\section{Consent for publication}

$\mathrm{NCl}$ has provided their consent to publish.

\section{Competing interests}

Not applicable to authors except MC, who is on the Board of Directors and an investor in Gibson Oncology, Inc., which has licensed indenoisoquinoline intellectual property owned by Purdue University. Neither Gibson Oncology nor any other commercial company sponsored or provided other direct financial support to the author or his laboratory for the research reported in this article.

\section{Publisher's Note}

Springer Nature remains neutral with regard to jurisdictional claims in published maps and institutional affiliations.

\section{Author details}

'Department of Cell Biology and Physiology, University of North Carolina School of Medicine, Neuroscience Research Building, Room 5119115 Mason Farm Rd., Campus Box 7545, Chapel Hill, NC 27599-7545, USA. ²Department of Medicinal Chemistry and Molecular Pharmacology, Purdue University School of Pharmacy and the Purdue Center for Cancer Research, West Lafayette, IN, USA. ${ }^{3}$ Developmental Therapeutics Branch and Laboratory of Molecular Pharmacology, Center for Cancer Research, National Cancer Institute, Bethesda, MD, USA. ${ }^{4}$ UNC Neuroscience Center, Carolina Institute for Developmental Disabilities, University of North Carolina School of Medicine, Chapel Hill, NC, USA.

Received: 14 March 2018 Accepted: 6 August 2018 Published online: 17 August 2018

\section{References}

1. Dagli Al, Mueller J, Williams CA. Angelman syndrome. In: Adam MP, Ardinger HH, Pagon RA, Wallace SE, LJH B, Mefford HC, Stephens K, Amemiya A, Ledbetter N, editors. GeneReviews(R). Seattle (WA):University of Washington; 1993.

2. Clayton-Smith J. Angelman syndrome: a review of the clinical and genetic aspects. J Med Genet. 2003;40(2):87-95.

3. Dan B. Angelman syndrome: current understanding and research prospects. Epilepsia. 2009:50(11):2331-9.

4. Williams CA. Neurological aspects of the Angelman syndrome. Brain Dev. 2005;27(2):88-94.

5. Mabb AM, Judson MC, Zylka MJ, Philpot BD. Angelman syndrome: insights into genomic imprinting and neurodevelopmental phenotypes. Trends Neurosci. 2011;34(6):293-303.

6. Steffenburg S, Gillberg CL, Steffenburg U, Kyllerman M. Autism in Angelman syndrome: a population-based study. Pediatr Neurol. 1996;14(2):131-6.

7. Dan B. Behavior and neuropsychiatric manifestations in Angelman syndrome. Neuropsychiatr Dis Treat. 2008;4;577.

8. Vu TH, Hoffman AR. Imprinting of the Angelman syndrome gene, UBE3A, is restricted to brain. Nat Genet. 1997;17(1):12-3.

9. Huang HS, Allen JA, Mabb AM, King IF, Miriyala J, Taylor-Blake B, Sciaky N, Dutton JW Jr, Lee HM, Chen $\mathrm{X}$, et al. Topoisomerase inhibitors unsilence the dormant allele of Ube3a in neurons. Nature. 2011;481(7380):185-9.

10. Pommier $Y$, Cushman M. The indenoisoquinoline noncamptothecin topoisomerase I inhibitors: update and perspectives. Mol Cancer Ther. 2009; 8(5):1008-14.

11. King IF, Yandava CN, Mabb AM, Hsiao JS, Huang HS, Pearson BL, Calabrese JM, Starmer J, Parker JS, Magnuson T, et al. Topoisomerases facilitate transcription of long genes linked to autism. Nature. 2013;501(7465):58-62.

12. Bomgaars $L$. The development of camptothecin analogs in childhood cancers. Oncologist. 2001;6(6):506-16.

13. Philpot BD, Thompson CE, Franco L, Williams CA. Angelman syndrome: advancing the research frontier of neurodevelopmental disorders. J Neurodev Disord. 2011;3(1):50-6.

14. Rodriguez-Galindo C, Poquette CA, Marina NM, Head DR, Cain A, Meyer WH, Santana VM, Pappo AS. Hematologic abnormalities and acute myeloid leukemia in children and adolescents administered intensified chemotherapy for the Ewing sarcoma family of tumors. J Pediatr Hematol Oncol. 2000:22(4):321-9.

15. Blaney SM, Phillips PC, Packer RJ, Heideman RL, Berg SL, Adamson PC, Allen JC, Sallan SE, Jakacki RI, Lange BJ, et al. Phase II evaluation of topotecan for pediatric central nervous system tumors. Cancer. 1996;78(3):527-31.

16. Feun L, Savaraj N. Topoisomerase I inhibitors for the treatment of brain tumors. Expert Rev Anticancer Ther. 2008;8(5):707-16.

17. Minturn JE, Janss AJ, Fisher PG, Allen JC, Patti R, Phillips PC, Belasco JB. A phase II study of metronomic oral topotecan for recurrent childhood brain tumors. Pediatr Blood Cancer. 2010;56(1):39-44.

18. El-Gizawy SA, Hedaya MA. Comparative brain tissue distribution of camptothecin and topotecan in the rat. Cancer Chemother Pharmacol. 1999:43(5):364-70.

19. Motl S, Zhuang Y, Waters CM, Stewart CF. Pharmacokinetic considerations in the treatment of CNS tumours. Clin Pharmacokinet. 2006:45(9):871-903.

20. Houghton PJ, Cheshire PJ, Hallman JD, Lutz L, Friedman HS, Danks MK, Houghton JA. Efficacy of topoisomerase I inhibitors, topotecan and irinotecan, administered at low dose levels in protracted schedules to mice 
bearing xenografts of human tumors. Cancer Chemother Pharmacol. 1995 36(5):393-403.

21. Stone JB, DeAngelis LM. Cancer-treatment-induced neurotoxicity--focus on newer treatments. Nat Rev Clin Oncol. 2016;13(2):92-105.

22. Soffietti R, Trevisan E, Ruda R. Neurologic complications of chemotherapy and other newer and experimental approaches. Handb Clin Neurol. 2014; 121:1199-218

23. Ioanoviciu A, Antony S, Pommier Y, Staker BL, Stewart L, Cushman M. Synthesis and mechanism of action studies of a series of norindenoisoquinoline topoisomerase I poisons reveal an inhibitor with a flipped orientation in the ternary DNA-enzyme-inhibitor complex as determined by X-ray crystallographic analysis. J Med Chem. 2005;48(15):4803-14.

24. Marchand C, Antony S, Kohn KW, Cushman M, loanoviciu A, Staker BL, Burgin $A B$, Stewart L, Pommier Y. A novel norindenoisoquinoline structure reveals a common interfacial inhibitor paradigm for ternary trapping of the topoisomerase I-DNA covalent complex. Mol Cancer Ther. 2006;5(2):287-95.

25. Staker BL, Feese MD, Cushman M, Pommier Y, Zembower D, Stewart L, Burgin AB. Structures of three classes of anticancer agents bound to the human topoisomerase I-DNA covalent complex. J Med Chem. 2005;48(7): 2336-45.

26. Mabb AM, Simon JM, King IF, Lee HM, An LK, Philpot BD, Zylka MJ. Topoisomerase 1 regulates gene expression in neurons through cleavage complex-dependent and -independent mechanisms. PLoS One. 2016;11(5): e0156439.

27. Antony S, Kohlhagen G, Agama K, Jayaraman M, Cao S, Durrani FA, Rustum YM, Cushman M, Pommier Y. Cellular topoisomerase I inhibition and antiproliferative activity by MJ-III-65 (NSC 706744), an indenoisoquinoline topoisomerase I poison. Mol Pharmacol. 2005;67(2):523-30.

28. Cushman M, Jayaraman M, Vroman JA, Fukunaga AK, Fox BM, Kohlhagen G, Strumberg D, Pommier Y. Synthesis of new Indeno[1, 2-c]isoquinolines: cytotoxic con-camptothecin topoisomerase I inhibitors. J Med Chem. 2000; 43(20):3688-98.

29. Fox BM, Xiao X, Antony S, Kohlhagen G, Pommier Y, Staker BL, Stewart L, Cushman M. Design, synthesis, and biological evaluation of cytotoxic 11 alkenylindenoisoquinoline topoisomerase I inhibitors and indenoisoquinoline -camptothecin hybrids. J Med Chem. 2003:46(15):3275-82.

30. Strumberg D, Pommier Y, Paull K, Jayaraman M, Nagafuji P, Cushman M. Synthesis of cytotoxic indenoisoquinoline topoisomerase I poisons. J Med Chem. 1999;42(3):446-57.

31. Kinders RJ, Hollingshead M, Lawrence S, Ji J, Tabb B, Bonner WM, Pommier Y, Rubinstein L, Evrard YA, Parchment RE, et al. Development of a validated immunofluorescence assay for gammaH2AX as a pharmacodynamic marker of topoisomerase I inhibitor activity. Clinical cancer research : an official journal of the American Association for Cancer Research. 2010;16(22):5447-57.

32. Antony S, Agama KK, Miao ZH, Takagi K, Wright MH, Robles Al, Varticovski L, Nagarajan M, Morrell A, Cushman M, et al. Novel indenoisoquinolines NSC 725776 and NSC 724998 produce persistent topoisomerase I cleavage complexes and overcome multidrug resistance. Cancer Res. 2007;67(21): 10397-405.

33. Nagarajan M, Morrell A, loanoviciu A, Antony S, Kohlhagen G, Agama K, Hollingshead M, Pommier Y, Cushman M. Synthesis and evaluation of indenoisoquinoline topoisomerase I inhibitors substituted with nitrogen heterocycles. J Med Chem. 2006;49(21):6283-9.

34. Kummar S, Chen A, Gutierrez M, Pfister TD, Wang L, Redon C, Bonner WM, Yutzy W, Zhang Y, Kinders RJ, et al. Clinical and pharmacologic evaluation of two dosing schedules of indotecan (LMP400), a novel indenoisoquinoline, in patients with advanced solid tumors. Cancer Chemother Pharmacol. 2016;78(1):73-81.

35. Jiang $Y H$, Armstrong D, Albrecht U, Atkins CM, Noebels JL, Eichele G, Sweatt JD, Beaudet AL. Mutation of the Angelman ubiquitin ligase in mice causes increased cytoplasmic p53 and deficits of contextual learning and long-term potentiation. Neuron. 1998;21(4):799-811.

36. Dindot SV, Antalffy BA, Bhattacharjee MB, Beaudet AL. The Angelman syndrome ubiquitin ligase localizes to the synapse and nucleus, and maternal deficiency results in abnormal dendritic spine morphology. Hum Mol Genet. 2008;17(1):111-8.

37. Beck DE, Agama K, Marchand C, Chergui A, Pommier Y, Cushman M. Synthesis and biological evaluation of new carbohydrate-substituted indenoisoquinoline topoisomerase I inhibitors and improved syntheses of the experimental anticancer agents indotecan (LMP400) and indimitecan (LMP776). J Med Chem. 2014;57(4):1495-512.
38. Kim Y, Lee HM, Xiong Y, Sciaky N, Hulbert SW, Cao X, Everitt Jl, Jin J, Roth BL, Jiang YH. Targeting the histone methyltransferase G9a activates imprinted genes and improves survival of a mouse model of Prader-Willi syndrome. Nat Med. 2017:23(2):213-22.

39. Carpenter AE, Jones TR, Lamprecht MR, Clarke C, Kang IH, Friman O, Guertin DA, Chang JH, Lindquist RA, Moffat J, et al. CellProfiler: image analysis software for identifying and quantifying cell phenotypes. Genome Biol. 2006; (10):R100.

40. Guo J, Holleran J, Schmitz JC, Czambel K, Beumer JH, Eiseman JL: Pharmacokinetics and pharmacodynamics of indenoisoquinoline LMP400 (indotecan) in BALB/c female mice bearing CT-26 colon tumors. [abstract]. Proceedings of the 106th Annual Meeting of the American Association for. Cancer Research 2015.

41. Pommier Y. Topoisomerase I inhibitors: camptothecins and beyond. Nat Rev Cancer. 2006:6(10):789-802

42. Pommier Y, Sun Y, Huang SN, Nitiss JL. Roles of eukaryotic topoisomerases in transcription, replication and genomic stability. Nat Rev Mol Cell Biol. 2016;17(November 2016):703-21.

43. Garst J. Safety of topotecan in the treatment of recurrent small-cell lung cancer and ovarian cancer. Expert Opin Drug Saf. 2007;6(1):53-62.

44. Fuchs C, Mitchell EP, Hoff PM. Irinotecan in the treatment of colorectal cancer. Cancer Treat Rev. 2006:32(7):491-503.

45. Antony S, Jayaraman M, Laco G, Kohlhagen G, Kohn KW, Cushman M, Pommier Y. Differential induction of topoisomerase I-DNA cleavage complexes by the indenoisoquinoline MJ-III-65 (NSC 706744) and camptothecin: base sequence analysis and activity against camptothecinresistant topoisomerases I. Cancer Res. 2003;63(21):7428-35.

46. Mabb AM, Kullmann PH, Twomey MA, Miriyala J, Philpot BD, Zylka MJ. Topoisomerase 1 inhibition reversibly impairs synaptic function. Proc Natl Acad Sci U S A. 2014;111(48):17290-5.

47. Solier S, Ryan MC, Martin SE, Varma S, Kohn KW, Liu H, Zeeberg BR, Pommier Y. Transcription poisoning by topoisomerase I is controlled by gene length, splice sites, and miR-142-3p. Cancer Res. 2013;73(15):4830-9.

48. Nagarajan M, Xiao X, Antony S, Kohlhagen G, Pommier Y, Cushman M. Design, synthesis, and biological evaluation of indenoisoquinoline topoisomerase I inhibitors featuring polyamine side chains on the lactam nitrogen. J Med Chem. 2003:46(26):5712-24

49. Cinelli MA, Reddy PV, Lv PC, Liang JH, Chen L, Agama K, Pommier Y, van Breemen RB, Cushman M. Identification, synthesis, and biological evaluation of metabolites of the experimental cancer treatment drugs indotecan (LMP400) and indimitecan (LMP776) and investigation of isomerically hydroxylated indenoisoquinoline analogues as topoisomerase I poisons. J Med Chem. 2012;55(24):10844-62.

Ready to submit your research? Choose BMC and benefit from:

- fast, convenient online submission

- thorough peer review by experienced researchers in your field

- rapid publication on acceptance

- support for research data, including large and complex data types

- gold Open Access which fosters wider collaboration and increased citations

- maximum visibility for your research: over $100 \mathrm{M}$ website views per year

At $\mathrm{BMC}$, research is always in progress.

Learn more biomedcentral.com/submissions 